\title{
van der Corput inequality for real line and Wiener-Wintner theorem for amenable groups
}

\author{
El AbDalaoui EL HOUCEIN*
}

ABSTRACT. We extend the classical van der Corput inequality to the real line. As a consequence, we obtain a simple proof of the Wiener-Wintner theorem for the $\mathbb{R}$-action which assert that for any family of maps $\left(T_{t}\right)_{t \in \mathbb{R}}$ acting on the Lebesgue measure space $(\Omega, \mathcal{A}, \mu)$, where $\mu$ is a probability measure and for any $t \in \mathbb{R}, T_{t}$ is measure-preserving transformation on measure space $(\Omega, \mathcal{A}, \mu)$ with $T_{t} \circ T_{s}=T_{t+s}$, for any $t, s \in \mathbb{R}$. Then, for any $f \in L^{1}(\mu)$, there is a single null set off which $\lim _{T \rightarrow+\infty} \frac{1}{T} \int_{0}^{T} f\left(T_{t} \omega\right) e^{2 i \pi \theta t} d t$ exists for all $\theta \in \mathbb{R}$. We further present the joining proof of the amenable group version of Wiener-Wintner theorem due to Ornstein and Weiss .

Keywords: van der Corput inequality, Wiener-Wintner theorem, joinings, amenable group.

2020 Mathematics Subject Classification: 11K06, 28D05, 47A35.

\section{INTRODUCTION}

In this paper, using our generalization of van der Corput inequality for the real line, we present a simple proof of Wiener-Wintner theorem for the flows. We further present the joining proof of the amenable groups version of it due to Ornstein and Weiss [13]. This accomplished by applying the Furstenberg joinings machinery. The classical Wiener-Wintner theorem [15] assert the following.

Theorem 1.1. Let $(\Omega, \mathcal{A}, \mu, T)$ be a dynamical system, where $\mu$ is a probability measure. Then, for any $f$ in $L^{1}(\mu)$, there is a set $\Omega^{\prime}$ of full measure such that for any $\omega \in \Omega^{\prime}$ the sums

$$
\frac{1}{N} \sum_{0}^{N-1} f\left(T^{n} \omega\right) z^{n}
$$

converge for all $z$ in the unit circle $C=\{z \in \mathbb{C}:|z|=1\}$.

The original proof can be found in [15]. Subsequently, Furstenberg in [6] obtain a joining proof of Wiener-Wintner theorem. Later, I. Assani [2], A. Below \& V. Losert [3] proved the stronger version of this theorem. This stronger version is due to Bourgain [4]. Theirs proofs is based on the Hellinger integral (known also as affinity principle). In [10], E. Lesigne generalize Wiener-Wintner theorem to the polynomial case. His proof is based on the Furstenberg's joinings technique. Afterwards, in [11], using van der Corput inequality and the spectral theory 
of skew products, he extended the stronger version of polynomial Wiener-Wintner theorem to the case of weak-wixing dynamical systems ${ }^{1}$.

In this paper, we extend van der Corput inequality to the continuous time and we give a simple proof of the flow version of Wiener-Wintner theorem. We further present the OrnsteinWeiss's joining of the amenable group version of this fundamental theorem in ergodic theory. The proof is based on Furstenberg's joinings machinery combined with the recent result of E. Lindenstrauss [12].

The plan of the paper is as follows. In Section 2, we state and prove the continuous van der Corput inequality and the flow version of Wiener-Wintner theorem. In section 3, we state and prove the amenable group version of Wiener-Wintner theorem.

\section{VAN DER CORPUT FOR REAL LINE}

In this section, we state our first main result.

Theorem 2.2 (van der Corput). Let $(u(t))_{t \in[0, T]}$ be an integrable complex valued function and $S \in$ $(0, T]$. Then

$$
\left|\int_{0}^{T} u(t) d t\right|^{2} \leq \frac{S+T}{S^{2}} \int_{0}^{S} \int_{0}^{S} \int_{0}^{T} u\left(t+s^{\prime}-s\right) \bar{u}(t) d s d s^{\prime} d t .
$$

Proof. We start by noticing that we have

$$
S \int_{0}^{T} u(t) d t=\int_{0}^{T+S} \int_{0}^{S} \tilde{u}(t-s) d s d t
$$

where $\tilde{u}$ stand for

$$
\tilde{u}(t)= \begin{cases}0 & \text { if } t \leq 0 \\ u(t) & \text { if } 0 \leq t \leq T \\ 0 & \text { if not. }\end{cases}
$$

Indeed, we have

$$
\begin{aligned}
\int_{0}^{T+S} \int_{0}^{S} \tilde{u}(t-s) d s d t & =\int_{0}^{S} \int_{-s}^{T+S-s} \tilde{u}(t) d t d s \\
& =\int_{0}^{S} \int_{0}^{T} u(t) d t d s \\
& =S \int_{0}^{T} u(t) d t
\end{aligned}
$$

Whence,

$$
S^{2}\left|\int_{0}^{T} u(t) d t\right|^{2}=\left|\int_{0}^{T+S} \int_{0}^{S} \tilde{u}(t-s) d s d t\right|^{2} .
$$

Now, applying Cauchy-Schwarz inequality, we obtain

$$
S^{2}\left|\int_{0}^{T} u(t) d t\right|^{2} \leq(T+S)\left(\int_{0}^{T+S}\left|\int_{0}^{S} \tilde{u}(t-s) d s\right|^{2} d t\right) .
$$

\footnotetext{
${ }^{1}$ Seven year after the first version of this note was written, M. Lacey and E. Terwilleger [9] produce an oscillation proof of the Hilbert version of Wiener-Wintner theorem.
} 
But

$$
\begin{aligned}
\int_{0}^{T}\left|\int_{0}^{S} \tilde{u}(t-s) d s\right|^{2} d t & =\int_{0}^{T} \int_{0}^{S} \int_{0}^{S} \tilde{u}(t-s) \overline{\tilde{u}}\left(t-s^{\prime}\right) d s d s^{\prime} \\
& =\int_{0}^{T} \int_{0}^{S} \int_{0}^{S} \tilde{u}(t-s) \overline{\tilde{u}}\left(t-s^{\prime}\right) d s d s^{\prime} d t \\
& =\int_{0}^{S} \int_{0}^{S} \int_{0}^{T} \tilde{u}\left(t+s^{\prime}-s\right) \overline{\tilde{u}}(t) d t d s d s^{\prime}
\end{aligned}
$$

Whence

$$
\left|\int_{0}^{T} u(t) d t\right|^{2} \leq \frac{S+T}{S^{2}} \int_{0}^{S} \int_{0}^{S} \int_{0}^{T} u\left(t+s^{\prime}-s\right) \bar{u}(t) d s d s^{\prime} d t .
$$

This achieve the proof of the theorem.

Theorem 2.3 (Limit version of continuous van der Corput theorem). Let $(u(t))_{t \in \mathbb{R}}$ be a bounded complex valued function. Then

$$
\begin{aligned}
& \limsup _{T \rightarrow \infty}\left|\frac{1}{T} \int_{0}^{T} u(t) d t\right|^{2} \\
\leq & \limsup _{S \rightarrow \infty} \frac{1}{S^{2}} \int_{0}^{S} \int_{0}^{S} \limsup _{T \rightarrow \infty} \frac{1}{T} \int_{0}^{T} u\left(t+s^{\prime}-s\right) \bar{u}(t) d s d s^{\prime} d t .
\end{aligned}
$$

Proof. Straightforward from Theorem 2.2.

Now, let us state the continuous version of Wiener-Wintner theorem.

Theorem 2.4 (Continuous version of Wiener-Wintner theorem). Let $\left(T_{t}\right)_{t \in \mathbb{R}}$ be a maps acting on the Lebesgue measure space $(\Omega, \mathcal{A}, \mu)$, where $\mu$ is a probability measure and for any $t \in \mathbb{R}, T_{t}$ is measurepreserving transformation on measure space $(\Omega, \mathcal{A}, \mu)$ with $T_{t} \circ T_{s}=T_{t+s}$, for any $t, s \in \mathbb{R}$. Then, for any $f \in L^{1}(\mu)$, there is a single null set off which

$$
\lim _{T \rightarrow+\infty} \frac{1}{T} \int_{0}^{T} f\left(T_{t} \omega\right) e^{2 i \pi \theta t} d t
$$

exists for all $\theta \in \mathbb{R}$.

We will assume without loss of generality that $\mu$ ergodic. Indeed, on can use the ergodic decomposition of $\mu$. So, it is sufficient to prove the following :

Theorem 2.5. For any $f$ in $L^{2}(\mu)$, there is a set $\Omega^{\prime}$ of full measure such that the sums

$$
\lim _{T \rightarrow+\infty} \frac{1}{T} \int_{0}^{T} f\left(T_{t} \omega\right) e^{2 i \pi \theta t} d t
$$

converge to 0 for all $\theta$ in $\mathbb{R}$, where $e^{2 \pi i \theta} \notin e(T)$ and $\omega \in \Omega^{\prime}$. $e(T)$ stand for the set of eigenvalue of the Koopman operator $U_{T}: g \mapsto g \circ T$.

Before proceeding to the proof of Theorem 2.5, let us notice that it suffices to prove it for a dense class of functions ( $L^{2}$ functions for instance). Indeed, put

$$
R(\omega, f)=\limsup _{T \longrightarrow+\infty}\left|\int_{0}^{T} f\left(T_{t}(\omega)\right) e^{2 \pi i t \theta} d t\right|
$$


and assume that $g$ in the dense class for which theorem holds. Then

$$
R(\omega, f)=R(\omega, f-g),
$$

and

$$
\mu\{\omega: R(\omega, f-g)>\epsilon\} \leq \frac{\|f-g\|_{1}}{\epsilon} .
$$

We thus get by the density of $L^{2}(\mu)$ in $L^{1}(\mu)$, that there exist $g$ in $L^{2}(\mu)$ such that : $\|f-g\|_{1}<\epsilon^{2}$. Then

$$
\mu\{\omega: R(\omega, f-g)>\epsilon\} \leq \epsilon .
$$

Since $\epsilon$ is arbitrary, we see $R(\omega, f)=0$ a.e., where the null set excluded is independent of $\theta$.

We start by recalling that by Bochner theorem, for any $f \in L^{2}(X)$, there exists a unique finite Borel measure $\sigma_{f}$ on $\mathbb{R}$ such that

$$
\widehat{\sigma_{f}}(t)=\int_{\mathbb{R}} e^{-i t \xi} d \sigma_{f}(\xi)=\left\langle U_{t} f, f\right\rangle=\int_{\Omega} f \circ T_{t}(\omega) \cdot \bar{f}(\omega) d \mu(\omega) .
$$

$\sigma_{f}$ is called the spectral measure of $f$. If $f$ is eigenfunction with eigenfrequency $\lambda$, then the spectral measure is the Dirac measure at $\lambda$.

We need also the following fundamental results from [1].

Theorem 2.6. Let $\left(\Omega, \mathcal{A}, \mu,\left(T_{t}\right)_{t \in \mathbb{R}}\right)$ be an ergodic dynamical flow. Then, for any $S>0$ and all $f, g \in L^{2}(X)$, for almost all $\omega \in \Omega$, we have

$$
\lim _{\tau \rightarrow+\infty} \frac{1}{\tau} \int_{0}^{\tau} f\left(T_{t+s} \omega\right) \cdot g\left(T_{t} \omega\right) d t=\int_{\Omega} f \circ T_{s} \cdot g d \mu
$$

uniformly for s in the interval $[-S, S]$.

This yields the exact result need it.

Corollary 2.1. Let $f \in L^{2}(\mu)$. There exist a full measure subset $\Omega_{f}$ of $\Omega$ such that, for any $\omega \in \Omega_{f}$ and any $s \in \mathbb{R}$, we have

$$
\lim _{\tau \rightarrow \infty} \frac{1}{\tau} \int_{0}^{\tau} f\left(T_{t+s} \omega\right) \cdot \bar{f}\left(T_{t} \omega\right) d t=\int_{X} f \circ T_{s} \cdot \bar{f} d \mu .
$$

Proof of Theorem 2.5. Let $f$ in $L^{\infty}(\mu)$ and $\omega \in \Omega_{f}$ as in Corollary 2.1, then we have

$$
\begin{aligned}
\lim _{\tau \rightarrow \infty} \frac{1}{\tau} \int_{0}^{\tau} f\left(T_{t+s} \omega\right) \cdot \bar{f}\left(T_{t} \omega\right) d t & =\int_{X} f \circ T_{s} \cdot \bar{f} d \mu \\
& \stackrel{\text { def }}{=}<f \circ T_{s}, f>.
\end{aligned}
$$

Put

$$
u(t)=f\left(T_{t} \omega\right) e^{2 \pi i t \theta},
$$

and apply further van der Corput's inequality (Theorem 2.2) to get

$$
\begin{aligned}
& \left|\frac{1}{\tau} \int_{0}^{\tau} f\left(T_{t} \omega\right) e^{2 \pi i t \theta} d t\right|^{2} \\
\leq & \frac{S+\tau}{\tau S^{2}} \int_{0}^{S} \int_{0}^{S} e^{2 \pi i\left(s-s^{\prime}\right) \theta} \frac{1}{\tau} \int_{0}^{\tau} f\left(T_{t+s-s^{\prime}}\right) \bar{f}\left(T_{t} \omega\right) d t d s d s^{\prime} .
\end{aligned}
$$


We thus deduce that for almost all $\omega$ and all $\theta \in \mathbb{R}$, we have

$$
\begin{aligned}
& \limsup _{\tau \rightarrow \infty}\left|\frac{1}{\tau} \int_{0}^{\tau} f\left(T_{t} \omega\right) e^{2 \pi i t \theta} d t\right|^{2} \\
\leq & \frac{1}{S^{2}} \int_{0}^{S} \int_{0}^{S} e^{2 \pi i\left(s-s^{\prime}\right) \theta}\left(\lim _{\tau \rightarrow \infty} \frac{1}{\tau} \int_{0}^{\tau} f\left(T_{t+s-s^{\prime}}\right) \bar{f}\left(T_{t} \omega\right) d t\right) d s d s^{\prime} .
\end{aligned}
$$

This combined with Corollary 2.1 gives

$$
\begin{aligned}
& \limsup _{\tau \rightarrow \infty}\left|\frac{1}{\tau} \int_{0}^{\tau} f\left(T_{t} \omega\right) e^{2 \pi i t \theta} d t\right|^{2} \\
\leq & \frac{1}{S^{2}} \int_{0}^{S} \int_{0}^{S}\left(\int_{\mathbb{R}} e^{2 \pi i\left(s-s^{\prime}\right)(\theta-\gamma)} d \sigma_{f}\left(s-s^{\prime}\right)\right) d s d s^{\prime},
\end{aligned}
$$

where $\sigma_{f}$ stand for the spectral measure of $f$. But, since

$$
\frac{1}{S^{2}} \int_{0}^{S} \int_{0}^{S} e^{2 \pi i\left(s-s^{\prime}\right)(\theta-\gamma)} d s d s^{\prime}=\left|\frac{1}{S} \int_{0}^{S} e^{2 \pi i s(\theta-\gamma)} d s\right|^{2}
$$

if $\theta \neq \gamma$, we have

$$
\lim _{S \rightarrow \infty} \frac{1}{S^{2}} \int_{0}^{S} \int_{0}^{S} e^{2 \pi i\left(s-s^{\prime}\right)(\theta-\gamma)} d s d s^{\prime}=0 .
$$

Whence, if $e^{2 \pi i \theta}$ is not a eigenvalue of $\left(T_{t}\right)$, we have

$$
\lim _{S \rightarrow \infty} \frac{1}{S^{2}} \int_{0}^{S} \int_{0}^{S}\left(\int_{\mathbb{R}} e^{2 \pi i\left(s-s^{\prime}\right)(\theta-\gamma)} d \sigma_{f}\left(s-s^{\prime}\right)\right) d s d s^{\prime}=0 .
$$

Since all the sums are bounded, we deduce from Lebesgue theorem that for almost all $\omega$, and for all $\theta$ in $\mathbb{R}$, where $e^{2 \pi i \theta} \notin e(T)$,

$$
\lim _{\tau \rightarrow \infty} \frac{1}{\tau} \int_{0}^{\tau} f\left(T_{t} \omega\right) e^{2 \pi i t \theta} d t=0
$$

and this finish the proof of the theorem.

\section{JOINING'S PROOF OF WIENER-WINTNER THEOREM FOR ACTION OF AMENABLE GROUP}

In this section, we deal with actions on Lebesgue spaces, that is, given a locally compact groupe $G$ and the a Lebesgue space $(X, \mathcal{A}, \mu)$, a $G$-action is a measurable mapping $G \times X \rightarrow X$, $(g, x) \mapsto g . x$, such that for all $g, h \in G, g .(h . x)=(g h) . x$ and $e . x=x$ for almost all $x \in X$ ( where $e$ is the identity in $G$ ). Furthermore, $T_{g}: x \mapsto g . x$ is measure -preserving for every $g \in G$. We will mainly concerned with $G$ which is amenable group ( locally compact second countable) or the subclass of locally compact abelian groups.

We recall that $G$ is an amenable group if for any compact $K \subset G$ and $\delta>0$ there is a compact set $F \subset G$ such that

$$
\mathrm{h}_{L}(F \Delta K F)<\delta \mathrm{h}_{L}(F),
$$

where $\mathrm{h}_{L}$ stand for the left Haar measure on $G$. It is well known that the amenability is equivalent to the existence of Følner sequence $\left(F_{n}\right)$, that is, $\left(F_{n}\right)$ is a sequence of compact subsets of $G$ for which for every compact $K$ and $\delta>0$, for all large enough $n$ we have that $F_{n}$ satisfy (3.1). 
Assume further that $\left(F_{n}\right)$ satisfy the so-called Shulman Condition, that is, for some $C>0$ and all $n$

$$
\mathrm{h}_{L}\left(\bigcup_{k \leq n} F_{k}^{-1} F_{n}\right) \leq C \cdot \mathrm{h}_{L}\left(F_{n}\right) .
$$

Under this assumptions, E. Lindenstrauss proved that the Birkhoff pointwise ergodic theorem holds, that is, then for any $f \in L^{1}(\mu)$, there is a $G$-invariant $f^{*} \in L^{1}(\mu)$ such that

$$
\lim \frac{1}{\mathrm{~h}_{L}\left(F_{n}\right)} \int_{F_{n}} f(g \omega) d \mathrm{~h}_{L}(g)=f^{*}(\omega) \text { a.e.. }
$$

To formulate the $G$-version of Wiener-Wintner theorem, we replace the group rotations by homomorphisms $\Theta$ from $G$ to a finite dimensional unitary group $U_{d}$. The canonical action in this case is given by $g . u=\Theta(g) . u, u \in U_{d}$ and $g \in G$. The invariant measure is the Haar measure on $U_{d}$. In this setting, we formulate the Wiener-Wintner theorem as follows:

Theorem 3.7 (Group version of Wiener-Wintner theorem). Let $G$ be an amenable group acting on a Lebesgue space $(\Omega, \mathcal{A}, \mu)$ and assume that $G$ satisfy Shulman condition. Let $f \in L^{\infty}(\mu)$. Then, there is a set $\Omega_{f}$ of full measure such for any $\omega \in \Omega_{f}$

$$
\frac{1}{h_{L}\left(F_{n}\right)} \int_{F_{n}} f(g \omega) \phi(\Theta(a) u) d h_{L}(g)
$$

converge for all finite dimensional unitary representation $\Theta$ of $G$ into $U_{d}($ all $d)$, all continuous function $\phi$ on $U_{d}$ and all $u \in U_{d}$. We further have that the limit on the orthocomplement of the space spanned by the finite dimensional invariant subspaces is zero.

Before proceeding to the proof let us recall some important tools.

A joining of two actions of the same group $\mathcal{X}=(X, \mathcal{A}, \mu, G)$ and $\mathcal{Y}=(Y, \mathcal{B}, \nu, G)$ is the probability measure $\lambda$ on $(X \times Y, \mathcal{A} \times \mathcal{B})$ which is invariant under the diagonal action of $G$ $(g .(x, y)=(g . x, g . y))$ and whose marginals on $(\mathcal{A} \times Y)$ and $(X \times \mathcal{B})$ are $\mu$ and $\nu$ respectively (i.e. if $A \in \mathcal{A}, \lambda(A \times Y)=\mu(A)$; and if $B \in \mathcal{B}, \lambda(X \times B)=\nu(B))$. The set of joinings is never empty (take $\mu \times \nu$ ). As we deal with Lebesgue spaces, a joining $\lambda$ of two ergodic $G$-actions $\mathcal{X}$ and $\mathcal{Y}$ has the property that there exists a Lebesgue space $\Omega$ and the probability $\Phi$ on $\Omega$ such that $\lambda=\int \lambda_{\omega} d \Phi(\omega)$, where $\lambda_{\omega}$ is ergodic (this is just the ergodic decomposition of $\lambda$, and as the marginals of $\lambda$ are ergodic a.e., $\lambda_{\omega}$ is joining). Therefore the set of ergodic joinings is never empty. ${ }^{2}$

Historically, joinings were introduced by H. Furstenberg in his paper [7] on disjointness. In particular, he defined the important notion of disjointness for $\mathbb{Z}$-action in the following way : $(X, \mathcal{A}, \mu, T)$ and $(Y, \mathcal{B}, \nu, S)$ is disjoint if the only joining between them is the product joining. In the case of $G$-action, we have the following definition.

Definition 3.1. Let $\mathcal{X}$ and $\mathcal{Y}$ be two actions of the same group $G$. $\mathcal{X}$ and $\mathcal{Y}$ are disjoint if the only joining between them is the product joining. We denote this disjointness by $\mathcal{X} \perp \mathcal{Y}$.

In the case of $\mathbb{Z}$-actions, H. Hahn \& W. Parry obtain in [8] that if two transformations have mutually singular maximal types, then they are disjoint. But, as for the joinings theory, the spectral theory of $\mathbb{Z}$-actions can be extended to the case of locally abelien $G$-actions. Therefore, we have the following group version of Hahn-Parry theorem.

Theorem 3.8 (Hahn \& Parry). If two $G$-actions $\mathcal{X}$ and $\mathcal{Y}$ have mutually singular maximal spectral types, then they are disjoint.

\footnotetext{
${ }^{2}$ see [5], for instance.
} 
Proof. Let recall that the spectral measure of a function $f \in L^{2}(X)$ under the operators $U_{g}$ (defined on $L^{2}(X)$ by $U_{g}(f)=f \circ T_{g}$ ) is the measure $\sigma_{f}$ on $\hat{G}$ (dual group of $G$, i.e., the set of all continuous characters of $G$ ), where its Fourier transform $\hat{\sigma}_{f}$ is given by $\hat{\sigma}_{f}(g)=\left\langle U_{g} f, f>\right.$. Now, we follows the proof given in [14]. In $X \times Y$ endowed with a joining measure $\lambda$, consider $f_{1} \in L^{2}(X)$ and $f_{2} \in L^{2}(Y)$ and consider $H_{f_{1}}$ the $L^{2}(\lambda)$ closure of the linear span of the functions $\left(U_{g}\left(f_{1}\right)-\int f_{1} d \mu\right) \times 1_{Y}, g \in G$. The projection of $1_{X} \times f_{2}$ on $H_{f_{1}}$ will have a spectral measure absolutely continuous with respect to the spectral type of $U_{g}$ on $L^{2}(X)$ and thus has to be 0 . Therefore $1_{X} \times f_{2} \perp\left(f_{1}-\int f_{1}\right) \times 1_{Y}$, and $\int f_{1}(x) f_{2}(y) d \lambda(x, y)=\int f_{1} d \mu \int f_{2} d \nu$.

From this theorem, we have the following.

Corollary 3.2. Let $\chi_{0}$ be a non trivial character and define the action of $G$ on torus $\mathbb{T}$ by $\left(g, e^{i x}\right) \mapsto$ $\chi_{0}(g) e^{i x}$. Assume that for any $n \in \mathbb{Z}$, the character $\chi_{0}^{n}$ define on $G$ by $g \mapsto \chi_{0}\left(g^{n}\right)$ is not eigenvalue of the $G$-action on $\mathcal{X}$. The $G$-action on $\mathbb{T}$ and the $G$-action on $\mathcal{X}$ are disjoint.

Proof. Let recall that $\chi_{0}$ is a eigenvalue of $G$-action if there exists a eigenfunction $f \in L^{2}(X, \mu)$ such that $f \circ T_{g}=\chi_{0}(g) f$. We deduce that the spectral measure of $f$ is $\|f\|_{2}^{2} \delta_{\chi_{0}}\left(\delta_{\chi_{0}}\right.$ is the Dirac measure on $\chi_{0}$ ). Since for any $n \in \mathbb{Z}, \chi_{0}^{n}$ is not eigenvalue of $G$-action on $\mathcal{X}$, we conclude that the maximal spectral types of this two $G$-actions are mutually singular. Now apply the Hahn-Parry theorem to complete the proof.

For the general case of amenable group which satisfy Shulman condition, we have the following lemma from [13].

Lemma 3.1. Let $U$ be the closure of $\Theta(G)$ in $U_{d}$. Then, if the product $(U, \Theta, G) \times(\Omega, \mathbf{A}, \mu, G)$ is ergodic then there is only on $G$-invariant measure on $U \times \Omega$ that projects onto $\mu$ on $\Omega$.

Proof of Theorem 3.7. We start by assuming without lost of generality that the action on $(\Omega, \mathbf{A}, \mu, G)$ is ergodic and by presenting the proof for the case when $G$ is locally Abelien group. Let $f \in L^{\infty}(\mu)$ and $\phi$ continuous function. Then, by the pointwise theorem, there is a set of full measure of $\omega$. Assume that $\omega$ is in this subset and let $\chi_{0} \in \hat{G}$ such $\chi_{0}$ is not eigenvalue. Then, the product $(U, \Theta, G) \times(\Omega, \mathbf{A}, \mu, G)$ is ergodic. Moreover, by taking a subsequence $\left(n_{k}\right)$, we can assume that

$$
\lim _{k \longrightarrow+\infty} \frac{1}{\mathrm{~h}_{L}\left(F_{n_{k}}\right)} \int_{F_{n_{k}}} f(g \omega) \phi(\Theta(a) u) d \mathrm{~h}_{L}(g)=\lambda(f \otimes \phi) .
$$

It follows that $\lambda$ is a joining and by Corollary $3.2, \lambda=d h \times \mu$. We end the proof by noticing that there is a countable of eigenvalue. The general case follows in the same manner by taking

$$
F(\omega)=\int \psi(u) I\left(u_{1} \omega\right) d u,
$$

where $I$ is a bounded invariant functions on $U \times \Omega$ and $\psi$ is any positive continuous function on $u$. Therefore, transforming $F$ by $g$ is the same as transforming $\psi$ by $\Theta(g)$. We thus have that a nonconstant $I$ will give rise to finite dimensional invariant subspaces for $G$ on $\Omega$. Moreover, by taking $(U, \Theta, G)$ not in the list of countable representations $\left(U_{j}, \Theta_{j}, G\right)$, the condition of Lemma 3.1 is satisfied and therefore as before the only joining is the product measure, and we are done.

Question 3.9. We ask on the possible extension of van der Corput inequality to the locally compact group and its application to produce a direct proof of the group version of Wiener-Wintner theorem.

\section{Acknowledgment.}

The author would like to express his thanks to Jean-Paul Thouvenot and E. Lesigne for a discussion on the subject. 


\title{
REFERENCES
}

[1] El H. El Abdalaoui: On the spectral type of rank one flows and Banach problem with calculus of generalized Riesz products on the real line, arXiv:2007.03684 [math.DS].

[2] I. Assani: Wiener-Wintner property of the helical transform, Ergod. Th. \& Dynam. Sys., 10 (1992), 185-194.

[3] A. Below, V. Losert: The weighted pointwise ergodic theorem and the individual ergodic theorem along subsequences, Trans. Amer. Math. Soc., 288 (1985), 307-345.

[4] J. Bourgain: Double recurrence and almost sure convergence, J. reine angew. Math., 404 (1990), 140-161.

[5] A. Deljunco, D. Rudolph: On ergodic actions whose self joining are graphs, Ergod. Th. \& Dynam. Sys., 7 (1987), 531557.

[6] H. Furstenberg: Stationary process and prediction theory, Ann. Math. Studies, 44 Princeton University Press, Princeton (1960).

[7] H. Furstenberg: Disjointness in ergodic theory, minimal sets and problem in diophantine approximation, Math. Sys. Theory, 1 (1960), 1-49.

[8] F. Hahn, W. Parry: Some characteristic properties od dynamical system with quasi-discrete spectrum, Math. Sys. Theory, 2 (1968), 179-190.

[9] M. Lacey, E. Terwilleger: A Wiener-Wintner theorem for the Hilbert transform, Ark. Mat., 46 (2) (2008), 315-336.

[10] E. Lesigne: Un théorème de disjonction de systèmes dynamiques et une généralisation du théorème egodique de WienerWintner, Ergod. Th. \& Dynam. Sys., 10 (1990), 513-521.

[11] E. Lesigne: Spectre quasi-discret et théorème egodique de Wiener-Wintner pour les polynômes, Ergod. Th. \& Dynam. Sys., 13 (1993), 767-784.

[12] E. Lindenstrauss: Pointwise ergodic theorem for amenable groups, Invent. Math., 146 (2) (2001), 259-295..

[13] D. Ornstein, B. Weiss: Subsequences ergodic theorems for amenable groups, Isr. J. Maths., 79 (1992), 113-127.

[14] J-P. Thouvenot: Some properties and applications of joinings in ergodic theory, In Ergodic Theory and its connections with Harmonic Analysis (Proc. of Alexandria conference), K. E. Petersen and I. Salama, Eds, L.M.S. lectures notes 205, Cambridge Univ. Press, Cambridge (1995), 207-235.

[15] N. Wiener, A. Wintner: Harmonic analysis and ergodic theory , Amer. J. Math., 63 (1941), 415-426.

\author{
El AbDalaoui EL HOUCEIN \\ UNIVERSITY OF ROUEN NORMANDY \\ DEPARTMENT OF MATHEMATICS \\ CNRS, LMRS UMR 60 85, SAINT-ETIENNE DE ROUVRAY, FRANCE \\ ORCID: 0000-0003-2005-1852 \\ E-mail address: elHoucein.elAbdalaoui@univ-rouen.fr
}

